International Journal of Pure and Applied Mathematics

Volume 93 No. 1 2014, 85-94

ISSN: 1311-8080 (printed version); ISSN: 1314-3395 (on-line version)

url: http://www.ijpam.eu

doi: http://dx.doi.org/10.12732/ijpam.v93i1.7

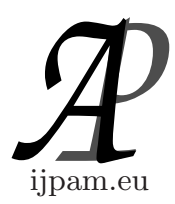

\title{
THREE-STEP ITERATIVE METHOD WITH EIGHTEENTH ORDER CONVERGENCE FOR SOLVING NONLINEAR EQUATIONS
}

\author{
Mohamed S.M. Bahgat ${ }^{1}$ §, M.A. Hafiz ${ }^{2}$ \\ ${ }^{1}$ Department of Mathematics \\ Faculty of Science \\ El-Minia University \\ EGYPT \\ ${ }^{1,2}$ Department of Mathematics \\ Faculty of Science and Arts \\ Najran University \\ Najran, 1988, SAUDI ARABIA
}

\begin{abstract}
In this paper, we propose and discuss a new higher-order iterative method for solving nonlinear equations. This method based on a Halley and Householder iterative method and using predictor-corrector technique. The convergence analysis of our method is discussed. It is established that the new method has convergence order eighteen. Numerical tests show that the new method is comparable with the well-known existing methods and gives better results.
\end{abstract}

AMS Subject Classification: 41A25, 65H05, 65K05

Key Words: nonlinear equations, convergence analysis, higher order convergence, iterative method

Received: November 9, 2013

(C) 2014 Academic Publications, Ltd. url: www.acadpubl.eu

$\S_{\text {Correspondence author }}$ 


\section{Introduction}

Finding iterative method for solving nonlinear equations is an important area of research in numerical analysis at it has interesting applications in several branches of pure and applied science can be studied in the general framework of the nonlinear equations $f(x)=0$ (see [2]). Due to their importance, several numerical methods have been suggested and analyzed under certain condition. These numerical methods have been constructed using different techniques such as Taylor series, homotopy perturbation method and its variant forms, quadrature formula (see [3]), variational iteration method, and decomposition method.For more details, see [1]-[14]. In this paper,based on a Halley and Householder iterative method and using predictor-corrector technique, we construct modification of Newton's method with higher-order convergence for solving nonlinear equations. The error equations are given theoretically to show that the proposed technique has eighteenth -order convergence. Commonly in the literature the efficiency of an iterative method is measured by the efficiency index defined as $I \approx p^{1 / d}$ (see [19]), where pis the order of convergence and dis the total number of functional evaluations per step. Therefore this method has efficiency index $18^{1 / 8} \approx 1.435$ which are higher than $2^{1 / 2} \approx 1.4142$ of the Steffensen's method (SM) (see [4], [16], $3^{1 / 4} \approx 1.3161$ of the (DHM) method [17]. Several examples are given to illustrate the efficiency and performance of this method.

\section{Iterative Method and Convergence Analysis}

Consider the nonlinear equation of the type

$$
f(x)=0
$$

For simplicity, we assume that $\alpha$ is a simply root of (2.1) and $\gamma$ is an initial approximation, sufficiently close to $\alpha$. Using the Taylor's series expansion of the function $f(x)$, we have

$$
f(\gamma)+(x-\gamma) f^{\prime}(\gamma)+\frac{(x-\gamma)}{2} f^{\prime \prime}(\gamma)=0
$$

Therefore

$$
x=\gamma-\frac{2 f(\gamma) f^{\prime}(\gamma)}{2 f^{\prime} 2(\gamma)-f(\gamma) f^{\prime \prime}(\gamma)}
$$

This equality allow us to suggest the following iterative method for solving the nonlinear equation (2.1). 
Algorithm 2.1. For a given $x_{0}$, find the approximate solution $x_{n+1}$ by the iterative scheme

$$
x_{n+1}=x_{n}-\frac{f\left(x_{n}\right)}{f^{\prime}\left(x_{n}\right)} .
$$

It is well known that Algorithm 2.1 has a quadratic convergence.

Algorithm 2.2. For a given $x_{0}$, compute approximates solution $x_{n+1}$ by the iterative scheme

$$
x_{n+1}=x_{n}-\frac{2 f\left(x_{n}\right) f^{\prime}\left(x_{n}\right)}{2 f^{\prime 2}\left(x_{n}\right)-f\left(x_{n}\right) f^{\prime \prime}\left(x_{n}\right)} .
$$

This is known as Halley's method has cubic convergence (see $[1,8]$ ).

Algorithm 2.3. For a given $x_{0}$, compute approximates solution $x_{n+1}$ by the iterative schemes

$$
x_{n+1}=x_{n}-\frac{f\left(x_{n}\right)}{f^{\prime}\left(x_{n}\right)}-\frac{f^{2}(x) f^{\prime \prime}\left(x_{n}\right)}{2 f^{\prime}\left(x_{n}\right)^{3}} .
$$

This is known as Householder's method and has cubic convergence, see [18]. Now we suggest and analyze a new three-step iterative method for solving the nonlinear equation. If we using classical Newton-Raphson method Algorithm 2.1 as a predictor and Algorithm 2.2 and Algorithm 2.3 as a corrector, which is the main motivation of this paper.

Algorithm 2.4. For a given $x_{0}$, compute approximates solution $x_{n+1}$ by the iterative schemes

$$
\begin{aligned}
w_{n} & =x_{n}-\frac{f\left(x_{n}\right)}{f^{\prime}\left(x_{n}\right)}, \\
y_{n} & =w_{n}-\frac{2 f\left(w_{n}\right) f^{\prime}\left(w_{n}\right)}{2 f^{\prime} 2\left(w_{n}\right)-f\left(w_{n}\right) f^{\prime \prime}\left(w_{n}\right)}, \\
x_{n+1} & =y_{n}-\frac{f\left(y_{n}\right)}{f^{\prime}\left(y_{n}\right)}-\frac{f^{2}(y) f^{\prime \prime}\left(y_{n}\right)}{2 f^{\prime}\left(y_{n}\right)^{3}}, \quad n=0,1,2, \ldots
\end{aligned}
$$

Algorithm 2.4 is called the predictor-corrector Newton-Halley method (PCNH) and has eighteen-order convergence. Let us now discuss the convergence analysis of Algorithm 2. 4.

Theorem 2.1. Let $r$ be a sample zero of sufficient differentiable function $f: \subseteq R \rightarrow R$ for an open interval $I$. If $x_{0}$ is sufficiently close to $r$, then 
the three-step iterative method defined by Algorithm 2.4 has eighteenth-order convergence.

Proof. Let $r$ be a simple zero of $f$. Since $f$ is sufficient differentiable, by expanding $f\left(x_{n}\right)$ and $f^{\prime}\left(x_{n}\right)$ about $r$, we get

$$
\begin{aligned}
f\left(x_{n}\right)=f(r)+\left(x_{n}-r\right) f^{\prime}(r)+\frac{\left(x_{n}-r\right)^{2}}{2 !} f^{(2)}(r) & +\frac{\left(x_{n}-r\right)^{3}}{3 !} f^{(3)}(r) \\
& +\frac{\left(x_{n}-r\right)^{4}}{4 !} f^{(4)}(r)+\cdots,
\end{aligned}
$$

then

$$
\begin{aligned}
f\left(x_{n}\right) & =f^{\prime}(r)\left[e_{n}+c_{2} e_{n}^{2}+c_{3} e_{n}^{3}+c_{4} e_{n}^{4}+\cdots\right], \\
f^{\prime}\left(x_{n}\right) & =f^{\prime}(r)\left[1+2 c_{2} e_{n}+3 c_{3} e_{n}^{2}+4 c_{4} e_{n}^{3}+\cdots\right],
\end{aligned}
$$

where $c_{k}=\frac{1}{k !} \frac{f^{(k)}(r)}{f^{\prime}(r)}, k=2,3, \ldots$, and $e_{n}=x_{n}-r$.

From (2.5) and (2.6), we have

$$
w_{n}=r+c_{2} e_{n}^{2}+2\left(c_{3}-c_{2}^{2}\right) e_{n}^{3}+\cdots,
$$

Let us set $W=w_{n}-r$. Then the equation (2.7) can be re-written in the form

$$
W=c_{2} e_{n}^{2}+2\left(c_{3}-c_{2}^{2}\right) e_{n}^{3}+\cdots,
$$

Now expanding $f\left(w_{n}\right), f^{\prime}\left(w_{n}\right), f^{\prime \prime}\left(w_{n}\right)$ about $r$ and using (2.7), we have

$$
\begin{aligned}
f\left(w_{n}\right) & =f(r)+\left(w_{n}-r\right) f^{\prime}(r)+\frac{\left(w_{n}-r\right)^{2}}{2 !} f^{(2)}(r)+\frac{\left(w_{n}-r\right)^{3}}{3 !} f^{(3)}(r)+\cdots \\
& =f^{\prime}(r)\left[W+c_{2} W^{2}+c_{3} W^{3}+c_{4} W^{4}+\cdots\right] \\
f^{\prime}\left(w_{n}\right) & =f^{\prime}(r)+\left(w_{n}-r\right) f^{\prime \prime}(r)+\frac{\left(w_{n}-r\right)^{2}}{2 !} f^{\prime \prime \prime}(r)+\cdots \\
& =f^{\prime}(r)\left[1+2 c_{2} W+3 c_{3} W^{2}+4 c_{4} W^{3}+\cdots\right] \\
f^{\prime \prime}\left(w_{n}\right) & =f^{\prime \prime}(r)+\left(w_{n}-r\right) f^{\prime \prime \prime}(r)+\cdots \\
& =f^{\prime}(r)\left[2 c_{2}+6 c_{3} W+\cdots\right] .
\end{aligned}
$$

Combining (2.7)-(2.11), we have

$$
y_{n}=r+\left(c_{2}^{2}-c_{3}\right) W^{3} .
$$


Also expanding $f\left(y_{n}\right), f^{\prime}\left(y_{n}\right), f^{\prime \prime}\left(y_{n}\right)$ in $r$ and using (2.12), we have

$$
\begin{aligned}
f\left(y_{n}\right)= & f^{\prime}(r)\left[\left(c_{2}^{2}-c_{3}\right) W^{3}+c_{2}\left(\left(c_{2}^{2}-c_{3}\right) W^{3}\right)^{2}\right. \\
& \left.+c_{3}\left(\left(c_{2}^{2}-c_{3}\right) W^{3}\right)^{3}+\cdots\right], \\
f^{\prime}\left(y_{n}\right)= & f^{\prime}(r)\left[1+2 c_{2}\left(\left(c_{2}^{2}-c_{3}\right) W^{3}\right)+3 c_{3}\left(\left(c_{2}^{2}-c_{3}\right) W^{3}\right)^{2}\right. \\
& \left.+4 c_{4}\left(\left(c_{2}^{2}-c_{3}\right) W^{3}\right)^{3}+\cdots\right], \\
f^{\prime \prime}\left(y_{n}\right)= & f^{\prime}(r)\left[2 c_{2}+6 c_{3}\left(\left(c_{2}^{2}-c_{3}\right) W^{3}\right)+12 c_{4}\left(\left(c_{2}^{2}-c_{3}\right) W^{3}\right)^{2}+\cdots\right] .
\end{aligned}
$$

Combining (2.12)-(2.15), we obtain

$$
\begin{aligned}
x_{n+1} & =r+\left(c_{2}^{2}-c_{3}\right) W^{3}-\left[\left(c_{2}^{2}-c_{3}\right) W^{3}+\left(-2 c_{2}^{2}+c_{3}\right)\left(\left(c_{2}^{2}-c_{3}\right) W^{3}\right)^{3}+\cdots\right] \\
& =r+\left(2 c_{2}^{2}-c_{3}\right)\left(c_{2}^{2}-c_{3}\right)^{3} W^{9}+\cdots \\
& =r+c_{2}^{9}\left(2 c_{2}^{2}-c_{3}\right)\left(c_{2}^{2}-c_{3}\right)^{3} e_{n}^{18}+O\left(e^{19}\right) .
\end{aligned}
$$

Finally, wehave

$$
e_{n+1}=c_{2}^{9}\left(2 c_{2}^{2}-c_{3}\right)\left(c_{2}^{2}-c_{3}\right)^{3} e_{n}^{18}+O\left(e^{19}\right),
$$

which shows that Algorithm 2.4 has eighteenth-order convergence.

Remark. The order of convergence of the iterative method 2.4 is 18. Per iteration of the iterative method 2.4 requires three evaluations of the function, three evaluation of first derivative, and two evaluation of second derivative. We take into account the definition of efficiency index (see [4], [15]), if we suppose that all theevaluations have the same cost as function one, we have that the efficiency index of the method 2.4 is $18^{1 / 8} \approx 1.435$, which is better $2^{1 / 2} \approx 1.4142$ of the Steffensen's method (SM) (see [16]), $3^{1 / 4} \approx 1.3161$ of the DHM method, see $[17]$.

\section{Numerical Examples}

For comparisons, we have used the fourth-order Jarratt method [19] (JM) and Ostrowski's method (OM) [15] defined respectively by

$$
\begin{aligned}
y_{n} & =x_{n}-\frac{2}{3} \frac{f\left(x_{n}\right)}{f^{\prime}\left(x_{n}\right)}, \\
x_{n+1} & =x_{n}-\left(1-\frac{3}{2} \frac{f^{\prime}\left(y_{n}\right)-f^{\prime}\left(x_{n}\right)}{3 f^{\prime}\left(y_{n}\right)-f^{\prime}\left(x_{n}\right)}\right) \frac{f\left(x_{n}\right)}{f^{\prime}\left(x_{n}\right)}
\end{aligned}
$$


and

$$
\begin{aligned}
y_{n} & =x_{n}-\frac{f\left(x_{n}\right)}{f^{\prime}\left(x_{n}\right)}, \\
x_{n+1} & =y_{n}-\frac{f\left(x_{n}\right)}{f\left(x_{n}\right)-2 f\left(y_{n}\right)} \frac{f\left(y_{n}\right)}{f^{\prime}\left(x_{n}\right)} .
\end{aligned}
$$

We consider here some numerical examples to demonstrate the performance of the new modified iterative method, namely (PCNH). We compare the classical Newton's method (NM), Jarratt method (JM), the Ostrowski's method $(\mathrm{OM})$ and $(\mathrm{PCNH})$. In the Table 1 and Table 2 the number of iteration is $n=$ 3 for all our examples. But in Table 1 our examples are tested with precision $\varepsilon=10^{-1000}$. The following stopping criteriais used for computer programs: $\left|x_{n+1}-x_{n}\right|+\left|f\left(x_{n+1}\right)\right|<\varepsilon$. The computational order of convergence (COC) can beapproximated using the following formula

$$
C O C \approx \frac{\ln \left|\left(x_{n+1}-x_{n}\right) /\left(x_{n}-x_{n-1}\right)\right|}{\ln \left|\left(x_{n}-x_{n-1}\right) /\left(x_{n-1}-x_{n-2}\right)\right|} .
$$

Table 1 shows the difference of the root $r$ and the approximation $x_{n}$ to $r$, where $r$ is the exact rootcomputed with 2000 significant digits, but only 25 digits are displayed for $x_{n}$. In Table 2, we listed the number of iterations for various methods. The absolute values of the function $f\left(x_{n}\right)$ and the computational order of convergence $(C O C)$ are also shown in Tables 2, 3. All the computations are performed using Maple, Version 15.The following examples are used for numerical testing:

$$
\begin{array}{lrrr}
f_{1}(x) & =x^{3}+4 x^{2}-10, & x_{0}=1 . f_{2}(x)=\sin ^{2} x-x^{2}+1, & x_{0}=1.3 . \\
f_{3}(x) & =x^{2}-e^{x}-3 x+2, & x_{0}=2 \cdot f_{4}(x)=\cos x-x, & x_{0}=1.7 . \\
f_{5}(x) & =(x-1)^{3}-1, & x_{0}=2.5 \cdot f_{6}(x)=x^{3}-10, & x_{0}=2 . \\
f_{7}(x) & =e^{x^{2}+7 x-30}-1, & x_{0}=3.1 . &
\end{array}
$$

Results are summarized in Tables 1, 2, 3 as it shows new algorithms are comparable with all of the methods and in most cases give better or equalresults.Table 2 Comparison of different methods.

\section{Conclusions}

In this paper, we have suggested and analyzed new higher-order iterative method and used for solving of nonlinear equations. This method based on a Halley 
Table 1: Comparison of Number of iterations forvarious methods required such that $\left|f\left(x_{n+1}\right)\right|<10^{-200}$.

\begin{tabular}{|l|l|l|l|l|l|l|l|}
\hline Method & $f_{1}$ & $f_{2}$ & $f_{3}$ & $f_{4}$ & $f_{5}$ & $f_{6}$ & $f_{7}$ \\
\hline Guess & 1 & 1.3 & 2 & 1.7 & 2.5 & 2 & 3.1 \\
\hline NM & 12 & 11 & 12 & 11 & 13 & 11 & 13 \\
\hline JM & 7 & 6 & 7 & 7 & 7 & 6 & 7 \\
\hline OM & 7 & 6 & 7 & 6 & 7 & 6 & 7 \\
\hline PCNH & 4 & 4 & 4 & 4 & 4 & 4 & 4 \\
\hline
\end{tabular}

Table 2: Comparison of different methods

\begin{tabular}{|c|c|c|c|c|c|}
\hline Method & $\mathrm{x}_{0}$ & $\mathrm{x}_{3}$ & $C O C$ & $\left|\mathbf{X}_{3}-\mathrm{X}_{2}\right|$ & $\left|f\left(x_{3}\right)\right|$ \\
\hline$f_{1}$ & 1 & & & & \\
\hline NM & & 1.3652366002021159462369662 & 1.88 & $3.66 \mathrm{E}-03$ & $1.09 \mathrm{E}-04$ \\
\hline $\mathrm{JM}$ & & 1.3652300134140968457610286 & 4.10 & $4.50 \mathrm{E}-12$ & $5.95 \mathrm{E}-46$ \\
\hline $\mathrm{OM}$ & & 1.3652300134140968457610286 & 4.10 & $4.50 \mathrm{E}-12$ & $5.95 \mathrm{E}-46$ \\
\hline $\mathrm{PCNH}$ & & 1.3652300134140968457610286 & 18.09 & $4.05 \mathrm{E}-225$ & $1.0 \mathrm{E}-1998$ \\
\hline $\mathrm{f}_{2}$ & 1.3 & & & & \\
\hline NM & & 1.4044916527111965739297374 & 1.98 & $7.57 \mathrm{E}-05$ & $1.12 \mathrm{E}-08$ \\
\hline JM & & 1.4044916482153412260350868 & 4.03 & $5.09 \mathrm{E}-18$ & $6.61 \mathrm{E}-70$ \\
\hline OM & & 1.4044916482153412260350868 & 4.03 & $5.96 \mathrm{E}-18$ & $1.29 \mathrm{E}-69$ \\
\hline$\overline{\mathrm{PCNH}}$ & & 1.4044916482153412260350868 & 18.03 & $1.69 \mathrm{E}-341$ & $9.0 \mathrm{E}-2000$ \\
\hline$f_{3}$ & 2 & & & & \\
\hline NM & & 0.2575292578013089584442857 & 7.68 & $3.31 \mathrm{E}-03$ & $3.88 \mathrm{E}-06$ \\
\hline $\mathrm{JM}$ & & 0.2575302854398607604553673 & 4.35 & $6.21 \mathrm{E}-06$ & $3.44 \mathrm{E}-23$ \\
\hline $\mathrm{OM}$ & & 0.2575302854398607604553673 & 4.55 & $8.79 \mathrm{E}-06$ & $1.02 \mathrm{E}-22$ \\
\hline $\mathrm{PCNH}$ & & 0.2575302854398607604553673 & 18.54 & $1.18 \mathrm{E}-133$ & $1.0 \mathrm{E}-1999$ \\
\hline $\mathrm{f}_{4}$ & 1.7 & & & & \\
\hline NM & & 0.7390851658032147634513238 & 1.53 & $3.84 \mathrm{E}-04$ & $5.45 \mathrm{E}-08$ \\
\hline $\mathrm{JM}$ & & 0.7390851332151606416553121 & 3.66 & $1.47 \mathrm{E}-12$ & $1.85 \mathrm{E}-49$ \\
\hline $\mathrm{OM}$ & & 0.7390851332151606416553121 & 3.67 & $3.34 \mathrm{E}-12$ & $5.32 \mathrm{E}-48$ \\
\hline$\overline{\mathrm{PCNH}}$ & & 0.7390851332151606416553121 & 17.61 & $2.25 \mathrm{E}-297$ & 0 \\
\hline$f_{5}$ & 2.5 & & & & \\
\hline NM & & 2.0003266792741527249601052 & 1.98 & $1.80 \mathrm{E}-02$ & $9.80 \mathrm{E}-04$ \\
\hline JM & & 2 & 3.73 & $2.55 \mathrm{E}-08$ & $8.43 \mathrm{E}-31$ \\
\hline $\mathrm{OM}$ & & 2 & 3.73 & $2.55 \mathrm{E}-08$ & $8.43 \mathrm{E}-31$ \\
\hline $\mathrm{PCNH}$ & & 2 & 17.69 & $3.63 \mathrm{E}-146$ & 0 \\
\hline
\end{tabular}


Table 3: Comparison of different methods

\begin{tabular}{|l|l|l|l|l|l|}
\hline Method & $\mathrm{x}_{0}$ & $\mathbf{x}_{3}$ & $C O C$ & $\left|\mathbf{x}_{3}-\mathbf{x}_{2}\right|$ & $\left|f\left(x_{3}\right)\right|$ \\
\hline $\mathrm{f}_{6}$ & 2 & & & & \\
\hline NM & & 2.1544346922369133091005011 & 1.97 & $6.89 \mathrm{E}-05$ & $3.07 \mathrm{E}-08$ \\
\hline $\mathrm{JM}$ & & 2.1544346900318837217592936 & 4.02 & $2.71 \mathrm{E}-19$ & $4.98 \mathrm{E}-75$ \\
\hline OM & & 2.1544346900318837217592936 & 4.02 & $2.71 \mathrm{E}-19$ & $4.98 \mathrm{E}-75$ \\
\hline PCNH & & 2.1544346900318837217592936 & 18.01 & $3.06 \mathrm{E}-370$ & 0 \\
\hline $\mathrm{f}_{7}$ & 3.1 & & & & \\
\hline NM & & 3.0007511637578020952127918 & 2.24 & $1.02 \mathrm{E}-02$ & $9.81 \mathrm{E}-03$ \\
\hline JM & & 3 & 3.91 & $1.46 \mathrm{E}-07$ & $6.17 \mathrm{E}-25$ \\
\hline OM & & 3 & 3.92 & $9.81 \mathrm{E}-08$ & $1.12 \mathrm{E}-25$ \\
\hline PCNH & & 3 & 17.74 & $8.84 \mathrm{E}-114$ & 0 \\
\hline
\end{tabular}

and Householder iterative method and using predictor-corrector technique. The error equations are given theoretically to show that the proposed technique haseighteenth-order convergence. The new method attain efficiency index of 1.435, which makes it competitive. In addition, the proposed method has been tested on a series of examples published in the literature and show good results when compared it with the previous literature.

\section{References}

[1] S.K. Khattri, Torgrim Log, Constructing third-order derivative-free iterative methods, Int. J. Comput. Math., 88, No. 7 (2011), 1509-1518, doi: 10.1080/00207160.2010.520705.

[2] I.K. Argyros, S.K. Khattri, An improved semi local convergence analysis for the Chebyshev method, Journal of Applied Mathematics and ComputingJuly, 42, No-s: 1,2 (2013), 509-528, doi: 10.1007/s12190-013-0647-3.

[3] S.K. Khattri, Quadrature based optimal iterative methods with applications in high-precision computing, Numer. Math. Theor. Meth. Appl., 5 (2012), 592-601.

[4] S.K. Khattri, Trond Steihaug, Algorithm for forming derivative-free optimal methods, Numerical Algorithms (2013), doi: 10.1007/s11075-0139715-x. 
[5] M.A. Hafiz, Solving nonlinear equations using steffensen-type methods with optimal order of convergence, Palestine Journal of Mathematics, 3, No. 1 (2014), 113-119.

[6] Mohamed S.M. Bahgat, M.A. Hafiz, New two-step predictor-corrector method with ninth order convergence for solving nonlinear equations, Journal of Advances in Mathematics, 2 (2013), 432-437.

[7] M.A. Hafiz, A new combined bracketing method for solving nonlinear equations, Journal of Mathematical and Computational Science, 3, No. 1 (2013).

[8] M.A. Hafiz, S.M.H. Al-Goria, Solving nonlinear equations using a new tenth-and seventh-order methods free from second derivative, International Journal of Differential Equations and Applications, 12, No. 3 (2013), 169183, 2013, doi: 10.12732/ijdea.v12i3.1344.

[9] M.A. Hafiz, M.S.M. Bahgat, Solving Nonlinear Equations using Two-Step Optimal Methods, Annual Review of Chaos Theory, Bifurcations and Dynamical, 2013.

[10] M.A. Hafiz, M.S.M. Bahgat, Solving nonsmooth equations using family of derivative-free optimal methods, Journal of the Egyptian Mathematical Society (2012).

[11] M.A. Hafiz, S.M.H. Al-Goria, New ninth-and seventh-order methods for solving nonlinear equations, European Scientific Journal, 8, No. 27 (2012).

[12] M.S.M. Bahgat, New two-step iterative methods for solving nonlinear equations, J. Math. Research, 4, No. 3 (2012), 128-131, doi: 10.5539/jmr.v4n3p128.

[13] M. Javidi, Fourth-order and fifth-order iterative methods for nonlinear algebraic equations, Math. Comput. Model., 50 (2009), 66-71.

[14] K.I. Noor, M.A. Noor, Predictor-corrector Halley method for nonlinear equations, Appl. Math. Comput., 188 (2007), 1587-1591.

[15] A.M. Ostrowski, Solutions of Equations and System of Equations, Academic Press, New York-London (1966).

[16] J.M. Ortega, W.G. Rheinboldt, Iterative Solutions of Nonlinear Equations in Several Variables, Press, New York- London, 1970, doi: $10.1137 / 1.9780898719468$. 
[17] M. Dehghan, M. Hajarian, Some derivative free quadratic and cubic convergence iterative formulaa for solving nonlinear equations, Comp \& App. Math., 29 (2010), 19-30, doi: 10.1590/S1807-03022010000100002.

[18] Khalida Inayat Noor, Muhammad Aslam Noor, Shaher Momani, Modified Householder iterative method for nonlinear equations. Appl. Math. Comput., 190 (2007), 1534-1539, doi: 10.1016/j.amc.2007.02.036.

[19] I.K. Argyros, D. Chen, Q. Qian, The Jarratt method in Banach space setting, J. Comput. Appl. Math., 51 (1994), 1-3, doi: 10.1016/03770427(94)90093-0. 